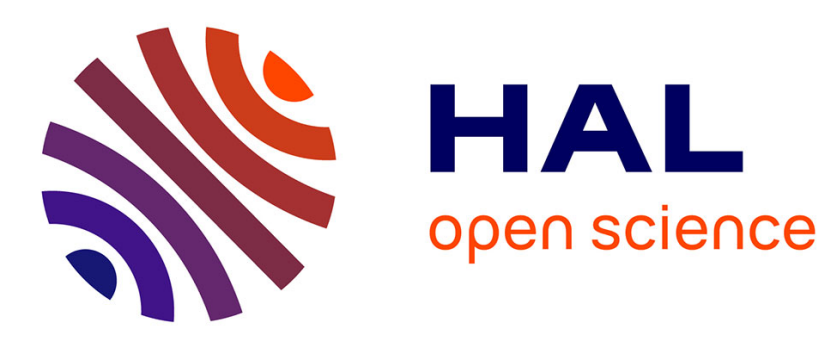

\title{
Note sur la construction des tubes à hydrogène
}

A. Cornu

\section{To cite this version:}

A. Cornu. Note sur la construction des tubes à hydrogène. J. Phys. Theor. Appl., 1886, 5 (1), pp.100-103. 10.1051/jphystap:018860050010001 . jpa-00238611

\section{HAL Id: jpa-00238611 https://hal.science/jpa-00238611}

Submitted on 1 Jan 1886

HAL is a multi-disciplinary open access archive for the deposit and dissemination of scientific research documents, whether they are published or not. The documents may come from teaching and research institutions in France or abroad, or from public or private research centers.
L'archive ouverte pluridisciplinaire HAL, est destinée au dépôt et à la diffusion de documents scientifiques de niveau recherche, publiés ou non, émanant des établissements d'enseignement et de recherche français ou étrangers, des laboratoires publics ou privés. 


\section{NOTE SUR LA CONSTRUCTION DES TUBES A HYDROGENE;}

Pir M. A. CORNU.

Tous les physiciens savent combien il est difficile d'obtenir des tubes à hydrogène ne contenant pas de substances étrangères : après de longs essais, j’ai réussi à obıenir à coup sûr des tubes ne contenant plus que des traces d'impurelés qu'on parviendrait même à faire disparaître entièrement en prolongeant les opérations.

Les précautions à prendre sont de plusieurs sortes :

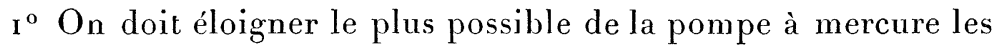
appareils que l'effluve électrique doit ou peut traverser; on y parvient en établissant la communication par l'intermédiaire de plusieurs mètres de tubes de verre courbés en hélice séparant des tubes de plus gros diamètres, contenant d'abord des fragments de

( $\left.{ }^{1}\right)$ D'autres séries de raies, de catégories voisines, semblent également satisfaire à la mème loi; néanmoins je me bornerai aujourd'húi à l'énoncé ci-dessus, bien que je risque ainsi de paraitre accorder aux raies spontanément renversables plus d'importance qu'elles n'en ont en réalité. 
soufre (aussi peu odorant que possible) et ensuite de la tournure de cuivre: le soufre arrête la vapeur de mercure et le cuivre arrête le soufre.

Pour isoler les extrémités de l'appareil, on interpose dans un des intervalles un baromètre dont la chambre a la forme d'un $\mathrm{V}$ et dont la cuvette est mobile pour former soupape de fermeture. Dans la capacité ainsi isolée on dispose un autre baromètre à large chambre présentant à son extrémité inférieure un tube latéral PR, presque capillaire, par lequel on introduit les gaz à raréfier.

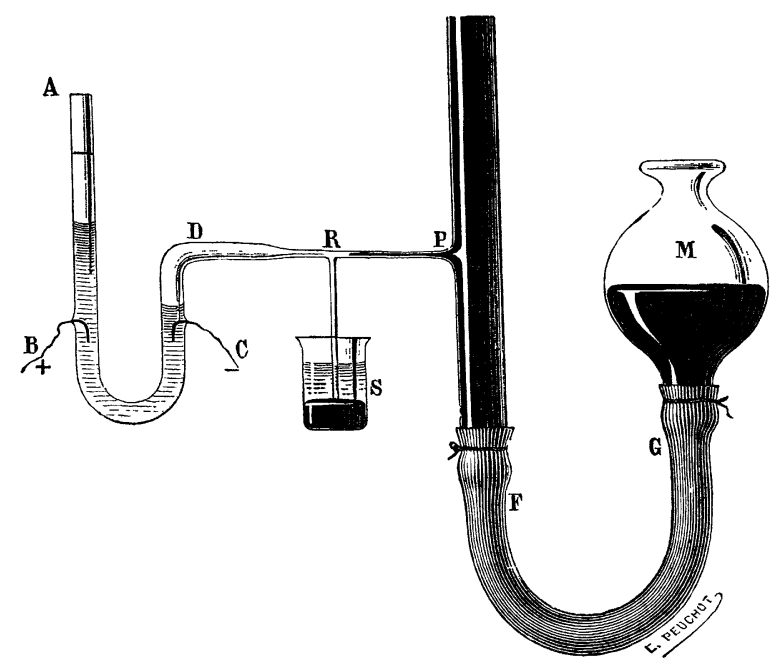

Il est à peine utile de dire que toutes les parties de l'appareil, jusqu'à la soupape à mercure, doivent être soudées et que les joints à caoutchouc, ou même les joints de verre rodés (réunis nécessairement avec une cire grasse), doivent être entièrement proscrits.

$2^{\circ}$ On prépare l'hydrogène par l'électrolyse de l'eau acidulée par l'acide phosphorique fondu; le voltamètre est formé d'un tube en $\mathrm{U}$, représenté en $A B C D$, raccordé par soudure au tube PR de jonction au baromètre. Au point de jonction $R$ est soudé un petit tube vertical plongeant dans un godet $\mathrm{S}$ contenant de l'eau acidulée et du mercure.

Pour faire passer une bulle de gaz dans le baromètre, et par 
suite dans la capacité isolée de l'appareil, il suffit de soulever avec précaution la cuvette mobile $M$ du baromètre, laquelle est formée d'un vase en forme de poire, réuni par un tube de caoutchouc.

On arrête le dégagement en abaissant la cuvette à un niveau convenable; si on l'abaissait trop, le mercure s'écoulerait dans le godet $\mathrm{R}$ comme par un siphon. Au commencement del'expérience on verse de l'eau acidulée dans le tube-voltamètre $\mathrm{ABCD}$; on le remplit totalement jusqu'au point $R$ et même jusqu'en $S$ en abaissant légèrement le godet $S$, parce que la branche RS fait siphon. Avec un peu d'adresse on arrive à chasser toute bulle d'air, soit en manœuvrant la cuvette mobile $\mathbf{M}$ du baromètre qui fait office de trompe, soit le niveau dans le tube AB.

Pour préparer le gaz, on fait passer le courant de quatre à cinq couples Bunsen par les fils B, C; l'un des gaz s'échappe par la branche ouverte BA, l'autre s'accumule dans la capacité DR: le tube RS fait alors office de tube de sûreté.

Pour obtenir le spectre de l'hydrogène exempt de substances étrangères, on commence par faire le vide, aussi complet que possible, dans l'appareil jusqu'à ce que l'étincelle d'induction ne passe plus dans les tubes à électrodes ou dans les tubes à gaines de M. Salet, qu'on emploie à l'étude spectrale. On fait passer quelques bulles d'hydrogène et l'on raréfie peu à peu. On voit apparaître inévitablement les bandes et les raies de divers composés carbonés : il suffit, en effet, que l'effluve électrique rencontre un tube mal nettoyé à l'intérieur ou la graisse d'un robinet pour que ces composés se forment instantanément (1). Pour se débarrasser de ces impuretés, on lave l'appareil avec de l'ozone; à cet effet, on renverse le courant dans le voltamètre après l'avoir vidé d'hydrogène, et l'on remplit l'appareil d'oxygène à la pression de $\mathbf{I}^{\mathrm{mm}}$

( $\left.{ }^{1}\right)$ J'ai observé ce phénomène pour la première fois dans des circonstances très instructives: le spectre de l'hydrogène étant assez pur, l'un des fils de la bobine d'induction vint à se détacher par hasard : aussitôt, tout l'appareil y compris la trompe, se remplit d'une lueur brillante rosée, mais, au bout de quelques secondes, cette lueur passa du rose au blanc: lorsque le fil de la bobine fut remis en place, le spectre de l'hydrogène était rempli de bandes et de raies étrangères. J'ai depuis répćté cette expérience qui, malheureusement, ne manque jamais de réussir. 
à $2^{\mathrm{mm}}$. On fait passer l'étincelle dans toute la capacité isolée par la soupape de mercure en fixant les fils de la bobine à deux lames d'étain collées extérieurement aux extrémités des tubes; on arrête de temps en temps l'étincelle pour faire le vide, qu'on pousse jusqu'à ce que l'effluve ne passe plus.

On recommence à remplir l'appareil avec quelques bulles d'hydrogène et on lave de même avec l'effluve d'hydrogène électrisé, et ainsi de suite alternativement avec l'hydrogène et l'oxygène.

On prolonge les opérations aussi longtemps que cela est nécessaire; à chaque fois on constate que le fond sur lequel se détachent les raies de l'hydrogène devient de plus en plus sombre : l'observation se fait avec un spectroscope ordinaire, ou mieux avec un spectroscope photographique qui donne des indications plus délicates en raison de l'intensité des raies des composés carbonés dans les régions violettes et ultra-violettes.

C'est en opérant ainsi que je suis parvenu à obtenir, avec des tubes à gaines ( $\left.{ }^{1}\right)$, les séries de spectres photographiques indiquées plus haut, et, bien que les dernières traces des impuretés fussent encore visibles, j'ai acquis la conviction qu'en prolongeant suffisamment les opérations on arriverait à les effacer entièrement. Dans ces tubes ainsi purifiés, l'éclat des raies de l'hydrogène est rraiment admirable. 